\title{
Reform on European union data protection rules in modern medicine
}

\author{
Andrey Kehayov \\ Faculty of Public Health, Medical University - Sofia
}

\begin{abstract}
Rapid technological developments and globalization have brought new challenges for the protection of personal data. The scale of the collection and sharing of personal data has increased significantly.

The right to the protection of personal data is not an absolute right and it must be considered in relation to its function in society and be balanced against other fundamental rights, in accordance with the principle of proportionality.

People shall be aware that disregard of the privacy and security of protected health information, confidential, personal or other sensitive information shall result in disciplinary action, up to and including dismissal.
\end{abstract}

\section{Keywords: Data protection, legal framework, European union}

The processing of personal data in relation to persons is protected as a fundamental right. The processing of personal data should be designed to serve human kind. The right to the protection of personal data needs to be considered in accordance to its function in society. This right needs to be balanced in accordance to other fundamental rights, in relation to the principle of proportionality.

Globalization and technological developments have brought new challenges for personal data protection. The level of protection of the rights and freedoms of persons with in relation to the processing of personal data should be equivalent in all countries within the European union. This ensures high level of protection of personal data and removes obstacles regarding the exchange of personal data within the European Union. 
The European Parliament, the Commission and the Council in December 2015 reached an agreement on setting up new rules for data protection for establishment of framework harmonizing regulations on data protection across the European union. The Council and the European Parliament adopted new Regulation and new Directive in April 2016.

The EU Regulation 2016/679 on the protection of natural persons with regard to the processing of personal data and on the free movement of such data and repeals Directive 95/46/EC. This Regulation applies to the processing of personal data by automated means, as well as to the processing of personal data other than by automated means, which form part of a filing system (1).

The definition of the term "personal data", under the Regulation, means any information relating to an identified or identifiable natural person. A person who can be identified, directly or indirectly is referred to as an identifiable natural person. The identification can be performed by reference to an identifier such as a name, an identification number, location data, an online identifier or to one or more factors specific to the physical, physiological, genetic, mental, economic, cultural or social identity of that natural person.

The new EU Regulation 2016/679 entered into force on 24 May 2016. It shall apply from 25 May 2018. Another European Union legal act related to the processing of personal data is a newly introduced Directive (2016/680). This Directive regulates the protection of natural persons with regard to the processing of personal data by competent authorities for the purposes of the prevention, investigation, detection or prosecution of criminal offences or the execution of criminal penalties, and on the free movement of such data. This legal act, which entered into force on 5 May 2016, is repealing Council Framework Decision 2008/977/JHA. All EU Member States have the obligation to transpose Directive 2016/680 into their national law by 6 May 2018 (2).

The new European Union framework is directly related to genetic data and to biometric data. Biometric data refers to personal data resulting from specific technical processing. This processing relates to the physical, physiological or behavioral characteristics of a natural person, allowing on confirming the identification of a natural person, which is unique and relays in facial images or dactyloscopic data.

Medical care also relates to genetic data, which is also sensitive data and represents personal data relating to the inherited or acquired genetic characteristics of a natural person. This data give unique information about the physiology or the health of a natural person and it result, in particular, from an analysis of a biological sample from the person in question.

Personal data, related to health, should include all data pertaining to the health status of a data subject and which reveals information relating to the past, current or future physical or mental health status of a person. This includes information about a person collected in the course of the registration for, or the provision of, health care services.

In relation to personal data protection, health services should be understood as referred to in Directive 2011/24/EU of the European Parliament and of the Council. Personal data concerning health includes information derived from the testing or examination of a body part or bodily substance, including from genetic data and biological samples and any information on, for example, a disease, disability, disease risk, medical history, clinical treatment or the physiological or biomedical state of a person. 
In order to exercise their right to access to healthcare, and to facilitate the patient mobility there is need of safety transmission of personal medical data through medical providers, which sometimes are situated in different countries. Patients claim to exercise their right to choose their own provider of healthcare services, sometimes beyond their national borders and especially when in need of medical treatment.

The confidentiality of patient records forms is central to the ethical tradition of medicine and health care. This tradition of confidentiality is in line with the requirements of data protection legal acts. Personal data must be obtained for a specified purpose, and must not be disclosed to any third party except in a manner compatible with that purpose.

In relation to the sensitivity of health-related information, it is imperative that professionals in this sector be clear about their use of personal data. The increasing role of health information technology platforms in organizing health information has led to the need to review the confidentiality, privacy, and security of electronic information.

Electronic health records (EHRs) provide a useful way to manage complex medical information, as such EHRs have been established to manage the large and complex datasets that accompany genetic/genomic tests and interpretations.

The inclusion of genetic/genomic information in EHRs should inform the determination of disease risk, appropriate drug dosing to avoid adverse events, and the selection of effective treatment. However, electronic health information is portable and mobile and the ease with which information can be disseminated through EHRs raises concern about the potential for unauthorized access to and use of this information (3).

Genetic information generally does not require more protection than other information that patients may view as sensitive (e.g., HIV status, mental health, or drug abuse). Over time, social norms may evolve so that mental health or HIV status is no longer viewed as sensitive, and perspectives regarding genetic/genomic test information may likewise evolve. The issue therefore becomes one of policy regarding access to sensitive health information.

Efforts related to selective and protective access to genetic/genomic test information must be combined with protective measures for other sensitive data, creating a consistent policy that applies to all sensitive health information (4).

People shall be aware that disregard of the privacy and security of protected health information, confidential, personal or other sensitive information shall result in disciplinary action, up to and including dismissal.

\section{References}

1. European Union, Regulation (EU) 2016/679 of the European parliament and of the Council on the protection of natural persons with regard to the processing of personal data and on the free movement of such data. 
2. European Union, Directive (EU) 2016/680 of the European parliament and of the Council of 27 April 2016 on the protection of natural persons with regard to the processing of personal data by competent authorities for the purposes of the prevention, investigation, detection or prosecution of criminal offences or the execution of criminal penalties, and on the free movement of such data.

3. Damschroder LJ, Pritts JL, Neblo MA, Kalarickal RJ, Creswell JW, Hayward RA. Patients, privacy and trust: patient's willingness to allow researchers to access their medical records. Soc Sci Med. 2007;64(1):223-235

4. Willison DJ, Emerson C, Szala-Meneok KV, Gibson E, Schwartz L, Weisbaum KM, et al. Access to medical records for research purposes: varying perceptions across research ethics boards. J Med Ethics. 2008;34(4):308-314.

\section{Corresponding author:}

Dr. Andrey Kehayov, MD, PhD

Faculty of Public Health

Medical University - Sofia

1527 Sofia, Bulgaria

8 Bialo more str. 